\title{
MULTIFUNCTIONAL ATOMIC FORCE MICROSCOPE CANTILEVER WITH LORENTZ FORCE ACTUATION AND SELF-HEATING FOR NANOMECHANICAL MEASUREMENTS IN AIR AND WATER

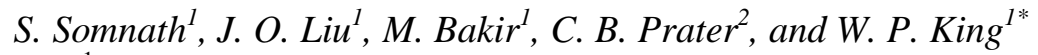 \\ ${ }^{1}$ University of Illinois, Urbana-Champaign, Illinois, USA \\ ${ }^{2}$ Anasys Instruments, Santa Barbara, California, USA
}

\begin{abstract}
This paper reports a multifunctional cantilever capable of selfheating and Lorentz force actuation for nanomechanical measurements over a large temperature range. The novel electrical design of the cantilever allows for high current to flow through the cantilever, resulting in high Lorentz force. The cantilever can selfheat beyond $800{ }^{\circ} \mathrm{C}$, and the heating is mostly independent of actuation force. When operated at its resonance frequency of 102 $\mathrm{kHz}$, the cantilever generates more than $5.3 \mu \mathrm{N}$ of Lorentz force while self-heating only to $44{ }^{\circ} \mathrm{C}$. This cantilever can generate about 7X larger Lorentz force than other heated cantilevers. The cantilever can be operated in air or water.
\end{abstract}

\section{INTRODUCTION}

The atomic force microscope (AFM) is a widely used tool for nanometer-scale measurements [1, 2]. Some characterization techniques oscillate the cantilever at resonance to acquire additional spectroscopic information and to maximize the signalto-noise. Cantilevers with integrated heater-thermometers can measure the temperature-dependence of material properties, and are widely used for materials characterization, especially for pharmaceuticals and composites [3]. However, heated cantilevers have not been designed for nanomechanical measurements and are limited in their ability to control and measure tip-sample forces due to parasitic resonances in piezoelectric cantilever actuation $[2,4$, 5].

Several strategies have been used to actuate AFM cantilevers, including photothermal excitation [6], integrated themomechanical elements [7], as well as piezoelectric [8] and electrostatic [9] actuation. However, these actuators are not suitable for actuating heated cantilevers due to the preexisting temperature distribution in the heated cantilever, and modifications necessary in the AFM hardware, and cantilever design. Electromagnetic actuation via Lorentz force [10] can actuate heated cantilevers with high signalto-noise without any modifications to the cantilever design or AFM hardware [4]. Most heated cantilevers have been developed for thermal topography sensing or data storage applications and are typically operated in contact mode [11-13]. Furthermore, heated cantilevers developed for tapping-mode were too small to generate sufficient Lorentz force or for operation in many commercial AFMs [5]. Thus, there is a need and an opportunity to design heated cantilevers optimized for Lorentz force actuation.

This paper reports the development of a Lorentz-thermal cantilever for nanomechanical measurements. We show how the novel cantilever design significantly increases the Lorentz force and allows independent control of heating and actuation compared to the state-of-art.

\section{CANTILEVER DESIGN}

Figure 1 shows the principle of the multifunctional Lorentzthermal cantilever. Alternating current passing through a U-shaped cantilever in the presence of a perpendicular magnetic field generates a Lorentz force at the cantilever free-end [4]. The magnitude of the Lorentz force is proportional to the strength of the magnetic field, the current, and the distance over which the current travels perpendicular to the magnetic field. Most of the heat generated in the cantilever heater flows into the substrate through the air, and this cantilever heat flow varies inversely with the distance between the cantilever and the substrate. The substrate topography is measured by tracking changes in the cantilever temperature [14]. Typically, heated cantilevers have a high resistance heater at the cantilever free-end [11]. The Lorentzthermal cantilever has low resistance Lorentz path in parallel with the high resistance heater to minimize the cantilever resistance.

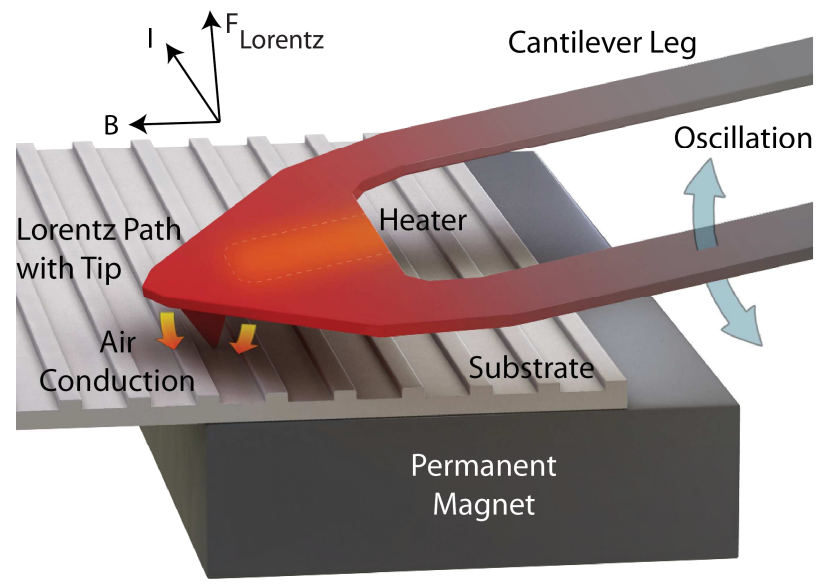

Figure 1: Working principles of Lorentz force cantilever actuation and thermal topography sensing. Alternating current flowing through the cantilever in the presence of a magnetic field generates a Lorentz force at the cantilever free-end that oscillates the cantilever. Heat flow from the cantilever varies with the cantilever-substrate distance. The substrate topography is measured by the cantilever temperature.

The Lorentz-thermal cantilever was designed to meet five main requirements. First, the cantilever must have a resonance frequency of at least $90 \mathrm{kHz}$ for tapping mode operation. Second, the cantilever must be capable of generating at least $2 \mu \mathrm{N}$ of Lorentz force. Third, it must be possible to operate the cantilever in a commercial AFM. Fourth, it must be possible to fabricate the cantilever using existing batch fabrication techniques [11]. Fifth, the cantilever should have thermal topography sensitivity that is comparable to that of the state-of-art Legacy cantilever $(0.3$ $\mathrm{mV} / \mathrm{nm}$ at $300^{\circ} \mathrm{C}$ ) [15].

Figure 2 illustrates how the Lorentz-thermal cantilever was designed to meet the performance goals. We developed finiteelement models using commercial software, COMSOL, to study how each design parameter affects the cantilever mechanical, electrical, and thermal characteristics. The cantilever length was shortened to achieve the desired resonance frequency, but this increased the cantilever stiffness. The cantilever stiffness was lowered by reducing the width of the cantilever legs, but a minimum leg width was maintained to ensure sufficient area for 
the AFM laser to reflect off the cantilever legs. Enlarging the distance between the cantilever legs increased the Lorentz force at the cost of the resonance frequency. We used a U-shaped cantilever geometry to localize the Lorentz force to the cantilever free-end. Since the cantilever was designed to have a $1 \mu \mathrm{m}$ tall tip, the cantilever free-end was tapered to ensure that the substrate contacted the tip instead of the cantilever body. The cantilever current was limited by the resistance of the cantilever legs, whose geometry was constrained by the mechanical performance goals of the cantilever. The cantilever current was maximized by enlarging the width of the Lorentz-path and shrinking the heater size. In contrast, the thermal topography sensitivity is maximized by increasing the heater size, which increases the heater resistance. Finally, the cantilever design was altered to account for the error and resolution of our photolithography system.

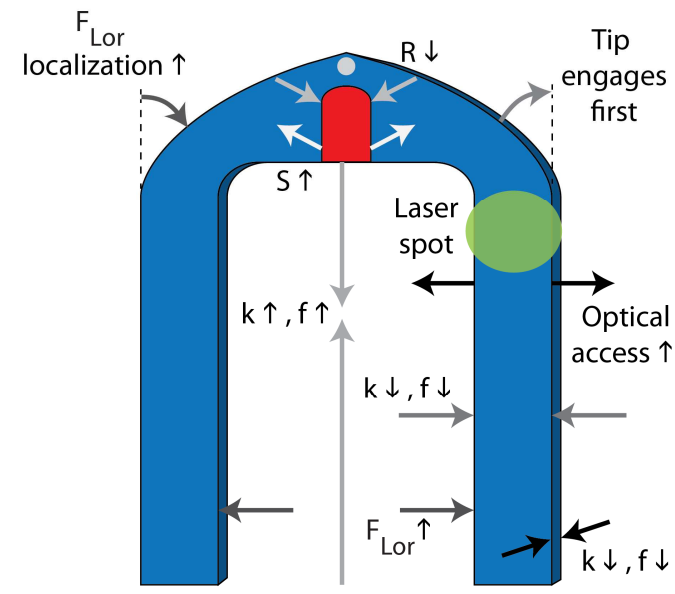

Figure 2: Illustration of how the cantilever geometry was varied to meet performance goals for the Lorentz-thermal cantilever. Our goal was to design a cantilever with resonance frequency $(f)$ at least $90 \mathrm{kHz}$, stiffness ( $k$ ) no more than $1.5 \mathrm{~N} / \mathrm{m}$, peak Lorentz. force $\left(F_{\text {Lor }}\right)$ at least $2 \mu N$, electrical resistance $(R)$ no more than $0.2 \mathrm{k} \Omega$, and thermal topography sensitivity $(S)$ of $0.3 \mathrm{mV} / \mathrm{nm}$ at 300 ${ }^{\circ} \mathrm{C}$.

\section{CANTILEVER CHARACTERIZATION}

Figure 3 shows a schematic and a scanning electron microscope (SEM) image of the fabricated Lorentz-thermal cantilever. The cantilever legs are short for tapping mode operation and $50 \mu \mathrm{m}$ apart to maximize Lorentz force. The cantilever has a 1 $\mu \mathrm{m}$ tall tip with $10 \mathrm{~nm}$ apex radius at the cantilever free-end. The cantilever has a stiffness of $1.7 \mathrm{~N} / \mathrm{m}$, resonance frequency of 102 $\mathrm{kHz}$, and electrical resistance of $0.17 \mathrm{k} \Omega$. The Lorentz-thermal cantilever was batch fabricated using the same procedure used for fabricating Legacy cantilevers. Table 1 compares the geometry and the characteristics of the Lorentz-thermal and Legacy cantilevers.
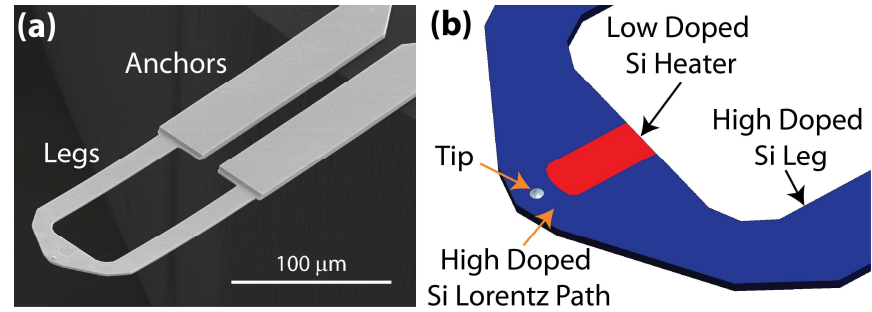

Figure 3: (a) Scanning Electron Microscope image and (b) schematic of the Lorentz-thermal cantilever. The cantilever freeend consists of two parallel current paths - a high resistance path through the heater and a low resistance Lorentz path around the heater.

Table 1: Comparison of cantilever geometry, characteristics and performance for Lorentz-thermal and Legacy cantilevers.

\begin{tabular}{lcc}
\hline & Legacy & Lorentz \\
\hline Geometry: & 167 & 130 \\
Cantilever Length $(\mu \mathrm{m})$ & 20 & 15 \\
Leg width $(\mu \mathrm{m})$ & 1 & 1.5 \\
Thickness $(\mu \mathrm{m})$ & 20 & 50 \\
$\begin{array}{l}\text { Distance between legs }(\mu \mathrm{m}) \\
\text { Heater area }\left(\mu \mathrm{m}^{2}\right)\end{array}$ & 137 & 102 \\
& & \\
Characteristics: & 2.2 & 0.17 \\
$\begin{array}{l}\text { Electrical resistance }(\mathrm{k} \Omega) \\
\text { Resonance Frequency }(\mathrm{kHz})\end{array}$ & 64 & 108 \\
$\begin{array}{l}\text { Stiffness }(\mathrm{N} / \mathrm{m}) \\
\text { Mechanical performance: }\end{array}$ & 0.53 & 1.71 \\
$\begin{array}{l}\text { Peak amplitude }(\mu \mathrm{m}) \\
\text { Peak force }(\mu \mathrm{N})\end{array}$ & 1.373 & $>2.779$ \\
$\begin{array}{l}\text { Amplitude improvement over } \\
\text { Legacy }\end{array}$ & 0.725 & $>5.309$ \\
$\begin{array}{l}\text { Force improvement over Legacy } \\
\text { Mean temperature rise per force } \\
\text { rise }\left({ }^{\circ} \mathrm{C} / \mu \mathrm{N}\right)\end{array}$ & 1 & $>2.03$ \\
\hline
\end{tabular}

Electrical and thermal characterization show that larger currents can flow through the Lorentz-thermal cantilever compared to the Legacy cantilever. The cantilevers were operated in series with a $1 \mathrm{k} \Omega$ current-limiting sense resistor. A Renishaw inVia Raman spectroscope measured the heater temperature as a function of DC heating voltage. Figure 4 shows the electrical and thermal properties of the two cantilevers. The cantilever current, resistance, and power vary non-linearly with the DC heating voltage in both cantilevers since the thermal conductivity and electrical resistivity of doped silicon vary with temperature [11]. Both cantilevers are capable of self-heating beyond $550{ }^{\circ} \mathrm{C}$. The Lorentz-thermal cantilever has electrical resistance which is almost an order of magnitude smaller than that of the Legacy cantilever. Consequently, the Lorentz-thermal cantilever allows more than 10 $\mathrm{mA}$ to pass through the cantilever which is higher than that of the Legacy cantilever $(1.2 \mathrm{~mA})$. The experimentally measured values are close to those from the simulation and the error is attributed to variations in the cantilever geometry and material properties due to limitations in the fabrication process. 


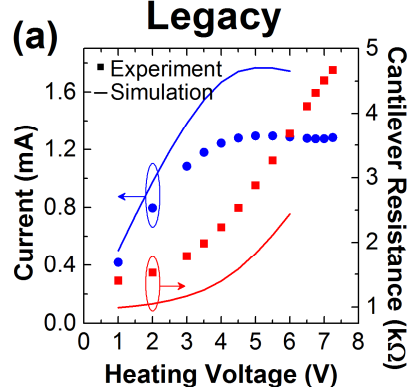

(c)

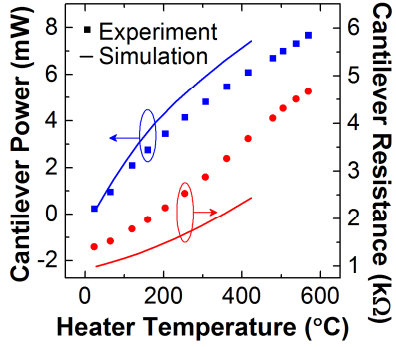

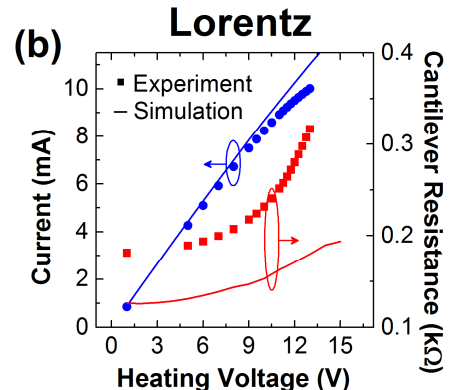

(d)

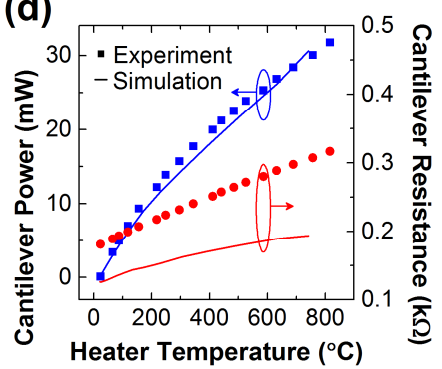

Figure 4: Electro-thermal characterization of Legacy and Lorentzthermal cantilevers. ( $a-b)$ Cantilever current and resistance as functions of DC heating voltage. (c-d) Cantilever power and resistance as functions of heater temperature.

The characteristics of cantilever actuation via Lorentz force and the piezoelectric actuator, used in typical AFMs, were compared. We mounted the cantilevers in an Asylum Research MFP 3D SA AFM. The cantilevers were positioned at the edge of a $\mathrm{NdFeB}$ cube magnet where the magnetic field is strongest [4]. A function generator supplied sinusoidal voltage to the piezoelectric actuator or the cantilever heating circuit for Lorentz force actuation. The cantilevers were operated in series with a $47 \mathrm{k} \Omega$ sense resistor to ensure a nearly pure sinusoidal current through the circuit [4]. The excitation frequency was increased from $100 \mathrm{~Hz}$ to $1 \mathrm{MHz}$ and the AFM optics recorded the cantilever oscillation amplitude. Figure 5 compares the cantilever response to piezoelectric and Lorentz force actuation schemes. The piezoelectric actuation shows numerous spurious peaks arising from resonances in the intermediate components between the actuator and the cantilever like the cantilever chip and cantilever holder. In contrast, Lorentz force actuation shows minimal noise and sharp peaks only at the cantilever harmonics since the force is applied directly at the cantilever free-end.

The mechanical and thermal performance of the Lorentzthermal cantilever was compared with that of the Legacy cantilever. [4]. A function generator increased the sinusoidal voltage to the cantilevers at the cantilever resonance frequency. Figure 6 shows the cantilever mechanical and thermal response to periodic current. The Lorentz force, oscillation amplitude and mean heater temperature increased with increasing periodic current. The lower resistance of the Lorentz-thermal cantilever enables larger current and Lorentz force $(5.3 \mu \mathrm{N})$ compared to the Legacy cantilever $(0.7 \mu \mathrm{N})$. With periodic current input, both cantilevers are capable of self-heating beyond $500{ }^{\circ} \mathrm{C}$ and the majority of the temperature rise occurs over a small range in cantilever current. The Lorentz thermal cantilever oscillated with larger amplitude $(2.8 \mu \mathrm{m})$ than the Legacy cantilever $(1.4 \mu \mathrm{m})$ despite having higher stiffness $(1.71 \mathrm{~N} / \mathrm{m})$ than the Legacy cantilever $(0.53 \mathrm{~N} / \mathrm{m})$. Due to the novel cantilever design the Lorentz-thermal cantilever self-heats only to $44{ }^{\circ} \mathrm{C}$ while

Legacy

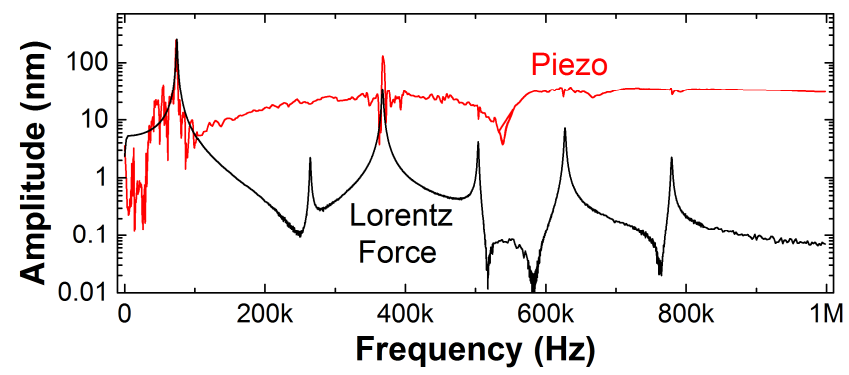

Lorentz

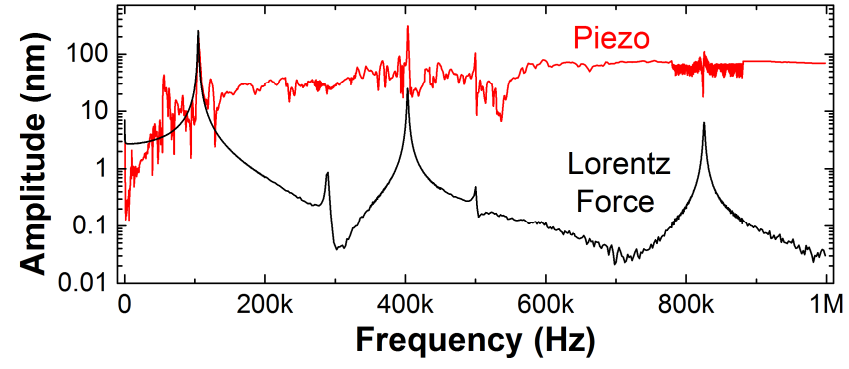

Figure 5: Cantilever oscillation amplitudes as functions of excitation frequency for piezoelectric and Lorentz force actuation. Piezoelectric actuation shows several parasitic peaks. Lorentz force actuation only shows amplitude peaks at the fundamental frequencies of the cantilever. (a)
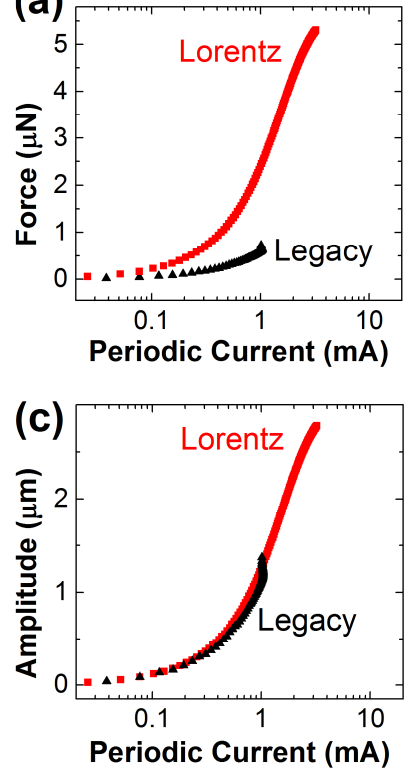
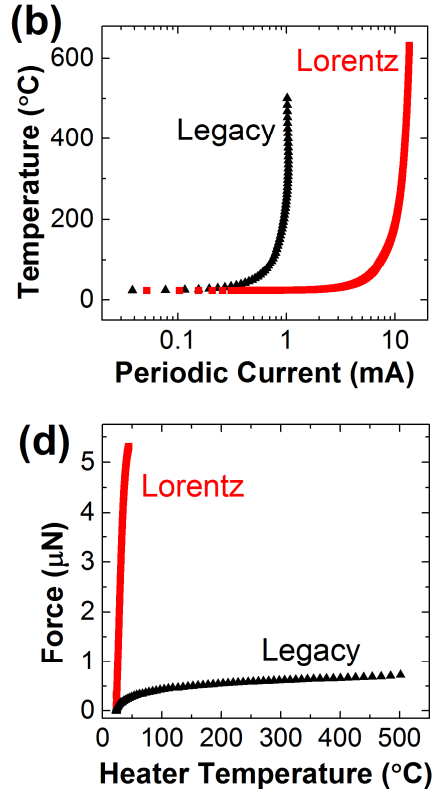

Figure 6: Cantilever response to periodic current for the Legacy and Lorentz-thermal cantilevers. (a) Generated Lorentz force, $(b)$ time-averaged heater temperature, and (c) cantilever amplitude as functions of the periodic current amplitude. (d) Lorentz-force as a function of heater temperature.

generating $5.3 \mu \mathrm{N}$ of force whereas the Legacy cantilever selfheats beyond $500{ }^{\circ} \mathrm{C}$ when generating $0.7 \mu \mathrm{N}$ Lorentz force. Thus, the Lorentz-thermal cantilever enables precise and independent control of Lorentz force and heater temperature via combinations of $\mathrm{AC}$ and $\mathrm{DC}$ excitation voltages. This capability is critical for 
accurate nanomechanical measurements over a large temperature range. Table 1 summarizes the performance of both cantilevers.

The Lorentz-thermal cantilever imaged a $100 \mathrm{~nm}$ tall silicon grating substrate in water to demonstrate the high signal-to-noise ratio of Lorentz force actuation and the ability to exert large actuation forces. The cantilever was submerged in a droplet of deionized water which was on the substrate. Lorentz force actuation clearly showed peaks at all the cantilever harmonics while it was impossible to actuate the cantilever using piezoelectric actuation. The cantilever scanned the grating in tapping mode via Lorentz force actuation at $1 \mu \mathrm{m} / \mathrm{sec}$. The cantilever was heated below $100{ }^{\circ} \mathrm{C}$ to prevent bubble formation. Figure 7 shows a topography image of the grating obtained from the cantilever thermal signal. The thermal signal had a noise-limited vertical resolution of $14 \mathrm{~nm}$. To our knowledge, this is the first demonstration of tapping mode operation via Lorentz force actuation and thermal topography sensing in a liquid medium.

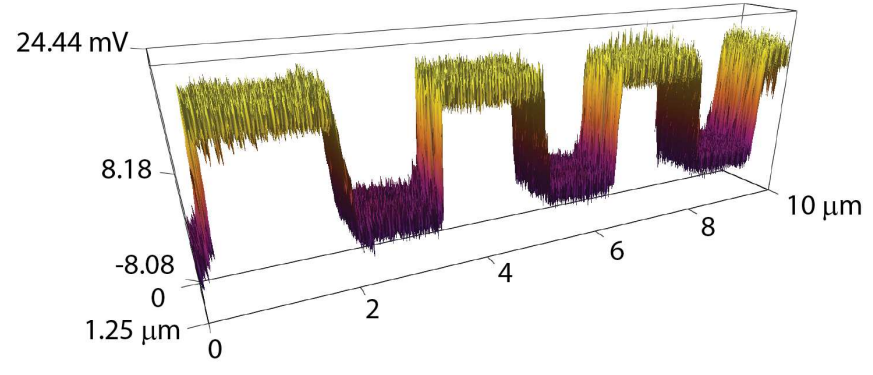

Figure 7: Topography image of a silicon grating acquired from the thermal signal of the Lorentz-thermal cantilever. The cantilever scanned the grating in tapping mode using Lorentz force actuation while being submerged in water.

\section{CONCLUSION}

This paper reports the design, characterization, and application of a multifunctional cantilever capable of Lorentz-force actuation and self-heating. The Lorentz-thermal cantilever uses a conductive path around the resistive heater that lowers the cantilever resistance to increase the cantilever current, and the Lorentz force. Lorentz force actuates the cantilever without introducing parasitic peaks since the force is applied directly at the cantilever free-end. The novel design of the Lorentz-thermal cantilever allows precise and independent control of Lorentz force (up to $5.3 \mu \mathrm{N}$ ) and self-heating (beyond $800{ }^{\circ} \mathrm{C}$ ). Overall this cantilever can generate about $7 \mathrm{X}$ larger Lorentz force compared to the state-of-art Legacy cantilever. This cantilever can be used for nanomechanical measurements using techniques such as contact resonance. Similar cantilever technology can be also be used to improve the sensitivity of microcantilever hotplates for thermogravimetry applications.

\section{ACKNOWLEDGEMENTS}

This work was supported by the NSF Center for ChemicalElectrical-Mechanical Manufacturing Systems, NSF CMMI 1068723, and DARPA TBN.

\section{REFERENCES}

[1] G. Binnig, C. Quate, and C. Gerber, "Atomic force microscope," Physical review letters, 56, 930-933, (1986).

[2] P. A. Yuya, D. C. Hurley, and J. A. Turner, "Contactresonance atomic force microscopy for viscoelasticity," Journal of Applied Physics, 104, 7, (2008).
[3] W. P. King, B. Bhatia, J. R. Felts, H. J. Kim, B. Kwon, B. Lee, et al., "Heated Atomic Force Microscope Cantilevers and their Applications," Annual Review of Heat Transfer, 16, 287-326, (2013).

[4] B. Lee, C. B. Prater, and W. P. King, "Lorentz force actuation of a heated atomic force microscope cantilever," Nanotechnology, 23, 055709, (2012).

[5] B. Lee, S. Somnath, and W. P. King, "Fast nanotopography imaging using a high speed cantilever with integrated heaterthermometer," Nanotechnology, 24, 135501, (2013).

[6] G. C. Ratcliff, D. A. Erie, and R. Superfine, "Photothermal modulation for oscillating mode atomic force microscopy in solution," Applied Physics Letters, 72, 1911-1913, (1998).

[7] J. Lee and W. P. King, "Microcantilever actuation via periodic internal heating," Review of Scientific Instruments, $78,-,(2007)$.

[8] T. Sulchek, R. Hsieh, J. D. Adams, S. C. Minne, C. F. Quate, and D. M. Adderton, "High-speed atomic force microscopy in liquid," Review of Scientific Instruments, 71, 2097-2099, (2000).

[9] H. Pozidis, W. Haberle, D. Wiesmann, U. Drechsler, M. Despont, T. R. Albrecht, et al., "Demonstration of thermomechanical recording at 641 Gbit/in(2)," IEEE Transactions on Magnetics, 40, 2531-2536, (2004).

[10] A. Buguin, O. Du Roure, and P. Silberzan, "Active atomic force microscopy cantilevers for imaging in liquids," Applied Physics Letters, 78, 2982-2984, (2001).

[11] J. Lee, T. Beechem, T. Wright, B. Nelson, S. Graham, and W. King, "Electrical, thermal, and mechanical characterization of silicon microcantilever heaters," Journal of Microelectromechanical Systems, 15, 1644-1655, (2006).

[12] B. W. Chui, T. D. Stowe, T. W. Kenny, H. J. Mamin, B. D. Terris, and D. Rugar, "Low-stiffness silicon cantilevers for thermal writing and piezoresistive readback with the atomic force microscope," Applied Physics Letters, 69, 2767-2769, (1996).

[13] H. Rothuizen, M. Despont, U. Drechsler, C. Hagleitner, A. Sebastian, and D. Wiesmann, "Design of power-optimized thermal cantilevers for scanning probe topography sensing," in IEEE MEMS 2009 Conference, Sorrento, Italy, 2009, pp. 603-606.

[14] K. Kim, K. Park, J. Lee, Z. Zhang, and W. King, "Nanotopographical imaging using a heated atomic force microscope cantilever probe," Sensors and Actuators A: Physical, 136, 95-103, (2007).

[15] S. Somnath, E. A. Corbin, and W. P. King, "Improved Nanotopography Sensing via Temperature Control of a Heated Atomic Force Microscope Cantilever," Sensors Journal, IEEE, 11, 2664-2670, (2011).

\section{CONTACT}

*W.P. King, tel: +1-217-244-3864; wpk@illinois.edu 